\title{
EXTERIOR POWERS AND TORSION FREE MODULES OVER DISCRETE VALUATION RINGS
}

\author{
BY
}

\author{
DAVID M. ARNOLD
}

\begin{abstract}
Pure $R$-submodules of the $p$-adic completion of a discrete valuation ring $R$ with unique prime ideal $(p)$ (called purely indecomposable $R$-modules) have been studied in detail. This paper contains an investigation of a new class of torsion free $R$-modules of finite rank (called totally indecomposable $R$-modules) properly containing the class of purely indecomposable $R$-modules of finite rank. Exterior powers are used to construct examples of totally indecomposable modules.
\end{abstract}

Introduction. $A$ torsion free (t.f.) $R$-module $A$ of finite rank is totally indecomposable if $A$ is reduced, $\operatorname{Hom}(A, R)=0$ (i.e. $A$ has no free summands) and every pure submodule of $A$ is either free or indecomposable. The p-rank of $A$ is the $R / p R$ dimension of $A / p A$.

Theorem 1.1. Suppose that $A$ is a reduced t.f. R-module of finite rank with $p$ rank $n$ and that $\operatorname{Hom}(A, R)=0$. The following statements are equivalent: (a) Every t.f. quotient of $A$ is either divisible or indecomposable; (b) $A$ is totally indecomposable; (c) Every pure submodule $B$ of $A$ with p-rank $B<n$ is a free $R$-module.

A t.f. $R$-module $A$ of finite rank is co-purely indecomposable (c.p.i.) if $A$ is reduced, $\operatorname{Hom}(A, R)=0$, and rank $A-p$-rank $A=1$. A consequence of [1] and Theorem 1.1 is that every c.p.i. module is totally indecomposable.

Theorem 1.7. If $A$ is a totally indecomposable $R$-module and rank $A \geq$ $2(p$-rank $A)-1$, then the endomorphism ring of $A$ is a local ring.

Theorem 1.7 has an immediate corollary: If $M=A_{1} \oplus \ldots \oplus A_{m}$, where each $A_{i}$ is totally indecomposable and rank $\left(A_{i}\right) \geq 2\left(p\right.$-rank $\left.A_{i}\right)-1$ for $1 \leq i \leq n$, then any two direct sum decompositions of $M$ have isomorphic refinements (Azumaya's theorem, e.g., see Lambek [8]).

Theorem 2.1. Assume that $A$ is a reduced t.f. R-module of finite rank with

Received by the editors July 15, 1971.

AMS 1970 subject classifications. Primary 20K 15; Secondary 16A10, 13C05, $15 A 75$.

Key words and phrases. Torsion free modules, finite rank, discrete valuation ring, purely indecomposable, local endomorphism ring. 
p-rank $n$ and that $\operatorname{Hom}(A, R)=0$. If the nth exterior power of $A$ is reduced, then $A$ is totally indecomposable.

If $A$ is totally indecomposable and if $0 \neq f \in E(A)$, the endomorphism ring of $A$, then $f$ is a monomorphism (Corollary 1.3).

Theorem 2.2. Suppose that $A$ is a reduced t.f. $R$-module of finite rank with $p$-rank $n$, that $\operatorname{Hom}(A, R)=0$, and that every $0 \neq f \in E(A)$ is a monomorphism. If f, $g \in E(A)$ and if $\bigwedge^{n} f=\bigwedge^{n} g$, then $f=r g$, where $r$ is an ntb root of unity of $R$.

Some applications of Theorem 2.2 to totally indecomposable modules are summarized in Corollary 2.3.

Theorem 2.1 and a slightly simplified version of the classical Kurosch matrix invariants are used to construct examples of totally indecomposable modules. Given positive integers $n$ and $k$, there is a t.f. $Z_{p}$-module $A$ with $p$-rank $n$, rank $n+k$ such that $\bigwedge^{n} A$ is reduced $\left(Z_{p}\right.$ is the localization of the ring of integers at a prime $p$ ). It is true (but we omit the proof) that the set of isomorphism classes of $Z_{p}$-modules, with $p$-rank $n$, rank $n+k$, such that $\bigwedge^{n} A$ is reduced, is uncountable.

The converses of Theorems 1.7 and 2.1 are, in general, false (Examples 3.2 and 3.3). Example 3.5 illustrates that the inequality, rank $A \geq 2(p$-rank $A)-1$, of Theorem 1.7 is best possible.

If $A$ and $B$ are c.p.i. modules of $p$-rank $n$, then $A$ and $B$ are quasi-isomorphic iff the $n$th exterior powers of $A$ and $B$ are isomorphic (e.g., see [1]). Example 3.4 illustrates that the analogous statement for totally indecomposable modules is, in general, false.

One can readily prove that a reduced t.f. $R$-module $A$ of finite rank is totally indecomposable iff every pure submodule of $A$ is either free or strongly indecomposable (i.e. has no quasi-direct summands). Consequently, Theorem 1.1 is true if the word "indecomposable" is replaced by "strongly indecomposable". Further, if $F$ is the duality given in [1], then $A$ is totally indecomposable iff $F A$ is totally indecomposable.

0 . Preliminaries. All modules are assumed to be t.f. $R$-modules unless otherwise specified. We write $\operatorname{Hom}(A, B)$ for $\operatorname{Hom}_{R}(A, B)$. Basic references are Kaplansky [7] and Rotman [9].

Every rank-1 torsion free module is isomorphic to either $R$ or $K$, the quotient field of $R$. A submodule $B$ of a module $A$ is pure if $p^{i} A \cap B=p^{i} B$ for all positive integers $i$ and reduced if $B$ has no divisible submodules. If $x$ is an element of the module $A$, the beight of $x$ in $A(b(x))$ is $i$ if $x \in p^{i} A \backslash p^{i+1} A$ and $\infty$ otherwise. 
A pure submodule $B$ of a module $A$ is a basic submodule of $A$ if $B$ is a free $R$-module and $A / B$ is divisible. Equivalently, $B$ is a free submodule of $A$ and $A / B$ is t.f. and divisible. Every t.f. module has a basic submodule and any two basic submodules of $A$ are isomorphic. If $B$ is a basic submodule of $A$, then $p$-rank $A=$ rank $B$.

Other properties of rank $(r)$ and $p$-rank $(r p)$ for t.f. modules of finite rank are: $r p(A)=0$ iff $A$ is divisible; $r p(A) \leq r(A) ; r p(A)=r(A)$ iff $A$ is free; if $0 \rightarrow$ $A \rightarrow B \rightarrow C \rightarrow 0$ is an exact sequence, then $r p(B)=r p(A)+r p(C)$ and $r(B)=$ $r(A)+r(C)$; and if $B \subset A$ and $A / B$ is torsion, then $r p(A) \leq r p(B)$ and $r(A)=r(B)$.

A t.f. $R$-module $A$ of finite rank with $p$-rank 1 is a purely indecomposable (p.i.) module iff $A$ is reduced. Furthermore, $E(A)$, the endomorphism ring of $A$, is a commutative local ring with $p E(A)$ as the ideal of nonunits.

1. Totally indecomposable modules.

Proof of Theorem 1.1. (a) $\Rightarrow$ (b) Assume that $B$ is a nonfree pure submodule of $A$, that $r p(B)<n$ and that $C$ is a basic submodule of $B$. Then $r p(A / C)>0$, since $r p(B)<n=r p(A)$. Note that $B / C$ is divisible, hence a summand of $A / C$. Therefore, $A / C$ is a nondivisible t.f. quotient of $A$ with a nonzero proper summand, a contradiction to (a).

Consequently, every nonfree pure submodule of $A$ has $p$-rank $n$. Let $B=$ $C \oplus D$ be a nonfree pure submodule of $A$. Then $r p(B)=r p(C)+r p(D)=n$, so $r p(C) \leq n$ and $r p(D) \leq n$. It now follows that either $C=0$ or $D=0$ since $C$ and $D$ are pure submodules of $A, A$ is reduced, and $B$ is not a free $R$-module.

(b) $\Rightarrow$ (c) Suppose that $C$ is a pure submodule of $A$ with $r p(C)<n$ and that $B$ is a basic submodule of $C$. There is a nonzero element $x+B$ of $A / B$ such that $x+B$ has zero height in $A / B$, since $r p(C)<n=r p(A)$. Therefore, $R(x+B)$ is a pure submodule of $A / B$ and $B \oplus R x$ is a pure submodule of $A$.

We prove that $C \oplus R x$ is a pure submodule of the totally indecomposable module $A$, which implies that $C \oplus R x$, hence $C$, is a free $R$-module. Clearly, $C \cap R x=0$. Assume that $a \in A$ and that $p^{i} a=c+r x \in C \oplus R x$. Since $C / B$ is divisible, there is $c^{\prime} \in C, b \in B$ with $c=p^{i} c^{\prime}+b$. Thus, $p^{i}\left(a-c^{\prime}\right)=b+r x \in$ $B \oplus R x$. Since $B \oplus R x$ is a pure submodule of $A, a-c^{\prime} \in B \oplus R x$ and $a \in C_{+}$ $(B \oplus R x) \subset C \oplus R x$.

(c) $\Rightarrow$ (a) Let $f: A \rightarrow C$ be an epimorphism, where $C$ is t.f. and not divisible, i.e. $r p(C)>0$. Then $r p(\operatorname{ker} f)<n$ so by (c), ker $f$ is free, i.e. $r p(\operatorname{ker} f)=$ $r(\operatorname{ker} f)$. Therefore, $r(C)-r p(C)=r(A)-r p(A)=k$. Suppose that $C=C_{1} \oplus C_{2}$, where $r p\left(C_{1}\right)>0$. Define $g: A \rightarrow C_{1}$ to be the composite of $f$ and the projection of $C$ onto $C_{1}$. By the preceding remarks, ker $g$ is free and $r\left(C_{1}\right)-r p\left(C_{1}\right)=$ $k$. But $k=r(C)-r p(C)=r\left(C_{1}\right)-r p\left(C_{1}\right)+r\left(C_{2}\right)-r p\left(C_{2}\right)$, so $r\left(C_{2}\right)=r p\left(C_{2}\right)$. If $C_{2} \neq 0$, then $C_{2}$ is a free $R$-module contradicting the assumption that $\operatorname{Hom}(A, R)$ $=0$. Therefore, $C_{2}=0$. 
Corollary 1.2. If $A$ is totally indecomposable, then every nonfree pure sub. module and every nondivisible t.f. quotient of $A$ is totally indecomposable.

Corollary 1.3. Assume that $B$ is a reduced t.f. $R$-module with $\operatorname{Hom}(B, R)=$ 0 , and that $A$ is totally indecomposable. If $0 \neq f \in \operatorname{Hom}(B, A)$, then $r p(\operatorname{ker} f) \leq$ $r p(B)-r p(A)$. In particular, $r p(B)=r p(A)$ implies that $f$ is monic.

Proof. Let $C$ be the pure submodule of $A$ generated by $f(B)$. Since $\operatorname{Hom}(B, R)=0, f(B)$ and $C$ are not free $R$-modules. By Theorem 1.1, rp $(C)=$ $r p(A)$, and $r p(C) \leq r p(f(B))$ since $C / f(B)$ is torsion. Finally, $r p(\operatorname{ker} f) \leq r p(B)-$ $r p(A)$ since $r p(\operatorname{ker} f)+r p(f(B))=r p(B)$. The last statement of the corollary follows from the assumption that $B$ is reduced.

Corollary 1.4. Suppose that $A$ is a reduced t.f. $R$-module of finite rank with p-rank $n$ and that $\operatorname{Hom}(A, R)=0$. Then $A$ is totally indecomposable iff $R x_{1} \oplus$ $\cdots \oplus R x_{n}$ is a basic submodule of $A$ for every $R$-independent subset $\left\{x_{1}, \cdots, x_{n}\right\}$ of $A$ with $x_{i} \in A \backslash p A$ for $1 \leq i \leq n$.

Proof. $(\Rightarrow)$ Let $X=\left\{x_{1}, \cdots, x_{n}\right\} \subset A / p A$ be an $R$-independent subset of $A$. Then $B$, the pure submodule of $A$ generated by $X$, is free (Theorem 1.1). Moreover, $B=R x_{1} \oplus \cdots \oplus R x_{n}$ since $X \subset A \backslash p A$. Finally, $A / B$ is divisible since $r p(A / B)=r p(A)-r p(B)=0$.

$(\Leftarrow)$ Let $C$ be a pure submodule of $A$ with $l=r p(C)<n$. If $r(C) \geq n$, let $R x_{1} \oplus \cdots \oplus R x_{l}$ be a basic submodule of $C$ and choose $x_{l+1}, \cdots, x_{n} \in C \cap$ $(A \backslash p A)$ with $\left\{x_{1}, \cdots, x_{n}\right\}$ an $R$-independent subset of $A$. The hypotheses guarantee that $B=R x_{1} \oplus \cdots \oplus R x_{n}$ is a basic submodule of $A$. Consequently, $B$ is a basic submodule of $C$, contradicting the assumption that $r p(C)<n$.

Assume that $m=r(C)<n$, and choose $x_{m+1}, \cdots, x_{n} \in A$ such that $C^{\prime}$, the pure submodule of $A$ generated by $C$ and $\left\{x_{m+1}, \cdots, x_{n}\right\}$, has rank $n$. Then $r p\left(C^{\prime}\right)=n$, by the preceding remarks, so $C^{\prime}$ is a free $R$-module. Therefore, $C$ is free and $A$ is totally indecomposable by Theorem 1.1 .

Recall that $f$ is a unit in $E(A)$ iff $f$ is an automorphism of $A$.

Lemma 1.5. Let $A$ be a t.f. $R$-module of finite rank with p-rank $n$, and let $B$ be a basic submodule of $A$. Then $f$ is a unit in $E(A)$ iff $f$ is a monomorphism and $f(B)$ is a pure submodule of $A$.

Proof. $(\Rightarrow)$ Clear.

$(\Leftarrow)$ There is a commutative diagram

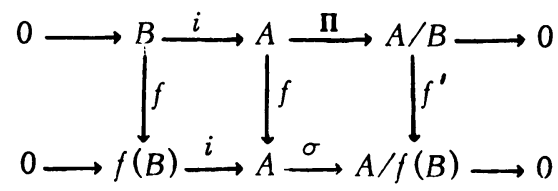


with exact rows where $i$ is an inclusion map, $\Pi$ and $\sigma$ are quotient maps and $f^{\prime}(a+B)=f(a)+f(B)$. Observe that $f^{\prime}$ is monic since $f$ is monic. Furthermore, $f(B)$ is a pure free submodule of $A$ of rank $n=p$-rank $A$ so $f(B)$ is a basic submodule of $A$. Therefore, $A / B$ and $A / f(B)$ are t.f. divisible $R$-modules of rank $k<\infty$ so $f^{\prime}$ is epic.

A routine diagram chase proves that $f$ is epic, hence $f$ is a unit in $E(A)$.

Define $A_{f}=\{x \in A \mid x=0$ or $x \neq 0$ and $b(f(x))>b(x)\}$, where $f \in E(A)$ and $b(x)$ is the height of $x$ in $A$, as defined in $\$ 0$. It is easy to prove that $A_{f}=0$ iff $f$ is a unit in $E(A)$.

Lemma 1.6. Suppose that $A$ is a totally indecomposable $R$-module of p-rank $n>1$, rank $n+k$, and that $f \in E(A)$.

(a) $A$ is a pure submodule of $A$;

(b) if $f$ is a nonunit of $E(A)$, then $\operatorname{rank}\left(A_{f}\right) \geq k+1$.

Proof. (a) The elementary properties of height suffice to prove that $R A, \subset A_{f}$ and that if $x \in A$ and if $p^{i} x \in A_{f}$, then $x \in A_{f}$.

Assume that $x, y \in A$, and that $x+y \neq 0$. If $R x \cap R y \neq 0$, then $r x=s y$ for some nonzero $r, s \in R$. Consequently, $x+y \in A_{f}$. Therefore, assume that $R x \cap$ $R y=0$. Then $B$, the pure submodule of $A$ generated by $x$ and $y$, is free (Theorem 1.1). In particular, $B=R x^{\prime} \oplus R y^{\prime}$ where $b\left(x^{\prime}\right)=b\left(y^{\prime}\right)=0$ and $p^{l} x^{\prime}=x$, $p^{m} y^{\prime}=y$. It now follows that $b(x+y)=\min (b(x), b(y))$.

Observe that $f$ is monic (Corollary 1.3) so $R x \cap R y=0$ implies that $R f(x)$ $\cap R f(y)=0$. Therefore, $b(f(x)+f(y))=\min (b(f(x)), b(f(y)))$ by the preceding remarks. Finally, $b(f(x)+f(y))=\min (b(f(x)), b(f(y)))>\min (b(x), b(y))$ so $x+y \in A_{f}$.

(b) Let $\left\{x_{1}, \cdots, x_{n+k}\right\}$ be a maximal $R$-independent subset of $A$. Write $x_{i}=p^{l i} y_{i}$, where $b\left(y_{i}\right)=0$ for $1 \leq i \leq n+k$. Then $\left\{y_{1}, \cdots, y_{n+k}\right\}$ is an $R$-independent subset of $A$ and $y_{i} \in A \backslash p A$ for $1 \leq i \leq n+k$.

Let $B_{1}=R y_{1} \oplus \cdots \oplus R y_{n}$, a pure submodule of $A$ by Corollary 1.4. By Lemma 1.5, there is $0 \neq z_{1} \in B_{1}$ such that $b\left(f\left(z_{1}\right)\right)>z_{1}$, i.e. $z_{1} \in A_{f}$. In fact, we may choose $z_{1}=y_{j}$, for some $j$.

Define $B_{i+1}=\left(B_{i} \oplus R y_{n+i}\right) / R z_{i}$ a basic submodule of $A$. Then there is $z_{i+1}=y_{j} \in A_{f}$, for some $y_{j} \notin R z_{1} \oplus \ldots \oplus R z_{i}$, by the preceding remarks. Consequently, $\left\{z_{1}, z_{2}, \cdots, z_{k+1}\right\}$ is an $R$-independent subset of $A_{f}$.

A ring $S$ is local if the nonunits of $S$ form an ideal of $S$, i.e. $S$ is local iff the sum of two nonunits of $S$ is again a nonunit of $S$.

Proof of Theorem 1.7. If $p$-rank $A=1$, then $E(A)$ is local. So assume that $p$-rank $A=n>1$, that $f$ and $g$ are nonunits of $E(A)$ and that $f+g=e$ is a unit of $E(A)$. Then rank $\left(A_{f}\right) \geq k+1$ and $\operatorname{rank}\left(A_{g}\right) \geq k+1$, where $p$-rank $A=n$, rank $A=n+k$ (Lemma 1.6(b)). Consider the exact sequence 


$$
0 \rightarrow A_{f} \cap A_{g} \rightarrow A_{f} \oplus A_{g} \stackrel{\phi}{\longrightarrow} A_{f}+A_{g} \rightarrow 0
$$

where $\phi(x+y)=x-y \in A$. Then $\operatorname{rank}\left(A_{f} \cap A_{g}\right) \geq 2 k+2-n-k \geq 1$. Since $A_{f}$ and $A_{g}$ are pure submodules of $A$ (Lemma 1.6.a), $A_{f} \cap A_{g}$ is a pure submodule of $A$. Choose $0 \neq x \in A_{f} \cap A_{g}$ with $x \in A \backslash p A$. Then $f(x) \in p A$ and $g(x) \in p A$, hence $(f+g)(x)=e(x) \in p A$. Therefore, $x \in A_{e}=0$ since $e$ is a unit of $E(A)$. This is a contradiction to the choice of $x$, thus $E(A)$ is local.

Note that if $f$ and $g$ are nonunits of $E(A)$, then $f+g=e$ is a unit of $A$ iff $A_{f} \cap A_{g}=0$. Example 3.5 demonstrates that this may happen if rank $A<$ $2(p$-rank $A)-1$.

2. Exterior powers. For $n \geq 2$, the $n t h$ exterior power of an $R$-module $A$, $\bigwedge^{n} A$, is given by $\bigwedge^{n} A=\left(\bigoplus^{n} A\right) / N$ where $N$ is the submodule of $\bigotimes^{n} A$ generated by $\left\{a_{1} \otimes \cdots \otimes a_{n} \mid a_{i}=a_{j}\right.$ for some $\left.i \neq j\right\}$. The $R$-module $\bigwedge^{n} A$ is generated by all elements of the form $a_{1} \wedge \cdots \wedge a_{n}=a_{1} \otimes \cdots \otimes a_{n}+N$. Define $\wedge^{\prime} A=A$ and $\bigwedge^{0} A=R$.

The following facts are used in the sequel: Let $A, B$, and $C$ be t.f. $R$-modules:

$$
\bigwedge^{n}(B \oplus C) \simeq \sum_{i=0}^{n} \oplus\left(\bigwedge^{i} B \otimes{ }^{n} \bigwedge^{i} C\right) \quad \text { (Bourbaki [3]) }
$$

(2) $\bigwedge^{n}$ is a functor: if $f: A \rightarrow B$,

$$
\wedge^{n} f\left(a_{1} \wedge \ldots \wedge a_{n}\right)=f\left(a_{1}\right) \wedge \ldots \wedge f\left(a_{n}\right) \quad(\text { Bourbaki [3] })
$$

(3) If $f: A \rightarrow B$ is monic, then $\bigwedge^{n} f: \bigwedge^{n} A \rightarrow \bigwedge^{n} B$ is monic (Flanders [4]);

(4) If $B$ is a pure (basic) submodule of $A$, then $\bigwedge^{n} B$ is isomorphic to a pure (basic) submodule of $\bigwedge^{n} A$. If $r(A)=l$ and $r p(A)=m$, then $r\left(\bigwedge^{n} A\right)=C_{l, n}$ and $r p\left(\bigwedge^{n} A\right)=C_{m, n}$ where $C_{i, j}$ is a binomial coefficient (Arnold [1]).

Proof of Theorem 2.1. Let $B$ be a pure submodule of $A$ with $m=r p(B)<n=$ $r p(A)$ and let $C=B \oplus E \subset A$ where $E=R x_{m+1} \oplus \cdots \oplus R x_{n}$. If $C$ is not free, then $\bigwedge^{m+1} C$ is a nonzero divisible $R$-module (by (4)). Furthermore, $\left(\bigwedge^{m+1} C\right) \otimes$ $\left(\bigwedge^{n-m-1} E\right)$ is isomorphic to a submodule of $\bigwedge^{n} A$ by (1) and (3). Now $r(E)=$ $n-m>0$, so $\bigwedge^{n-m-1} E$ is a nonzero $R$-module. Thus $\bigwedge^{m+1} C \otimes \bigwedge^{n-m-1} E$ is a nonzero divisible submodule of $\bigwedge^{n} A$, contradicting the assumption that $\bigwedge^{n} A$ is reduced.

Consequently, $C$ and $B$ are free $R$-modules. Now apply Theorem 1.1.

Theorem 2.2. Assume that $A$ is a reduced t.f. $R$-module of p-rank $n$, rank $n+k$, that $\operatorname{Hom}(A, R)=0$ and that every $0 \neq f \in E(A)$ is a monomorphism. If $f, g \in E(A)$ and if $\bigwedge^{n} f=\bigwedge^{n} g$, then $f=r g$, where $r$ is an nth root of unity of $R$.

Proof. Let $B=R x_{1} \oplus \cdots \oplus R x_{n}$ be a free submodule of $A$. If $f \neq 0$ and 
$g \neq 0$, then $f\left(x_{1}\right) \wedge \cdots \wedge f\left(x_{n}\right)=g\left(x_{1}\right) \wedge \cdots \wedge g\left(x_{n}\right) \neq 0$ since $\wedge^{n} f=\wedge^{n} g$ is a monomorphism (cited fact (3)). For $1 \leq j \leq n, f\left(x_{j}\right) \wedge g\left(x_{1}\right) \wedge \ldots \wedge g\left(x_{n}\right)=$ $f\left(x_{j}\right) \wedge f\left(x_{1}\right) \wedge \cdots \wedge f\left(x_{n}\right)=0 \in \wedge^{n+1} A$, so $\left\{f\left(x_{j}\right), g\left(x_{1}\right), \cdots, g\left(x_{n}\right)\right\}$ is an $R$-linearly dependent subset of $A$ (Bourbaki [3]). Consequently, $p^{l} f(B) \subset g(B)$ for some nonnegative integer $l$.

We prove that if $y \in A$, then there is a nonnegative integer $l_{y}$ and $0 \neq r_{y} \in R$ with $p^{l_{y}} f(y)=r_{y} g(y)$. Extend $y$ to an $R$-linearly independent subset $\left\{y, y_{1}, \cdots\right.$, $\left.y_{n}\right\}$ of $A$, observing that $\operatorname{rank} A>n$ since $A$ is not free. Define $C_{i}=R y \oplus R y_{1}$ $\oplus \cdots \oplus R y_{i-1} \oplus R y_{i+1} \oplus \cdots \oplus R y_{n}$ for $1 \leq i \leq n$. As a consequence of the preceding paragraph, there is a nonnegative integer $l$ with $p^{l} f\left(C_{i}\right) \subset g\left(C_{i}\right)$ for $1 \leq i$ $\leq n$. Furthermore, $R y=C_{1} \cap C_{2} \cap \ldots \cap C_{n}$. Thus, $p^{l} f(R y)=p^{l} f\left(C_{1} \cap C_{2} \cap \ldots \cap C_{n}\right)$ $C g\left(C_{1}\right) \cap g\left(C_{2}\right) \cap \cdots \cap g\left(C_{n}\right)=g(R y)$ (noting that $g$ is monic) and $p^{l} f(y)=$ $r_{y} g(y)$ for some $r_{y} \in R$.

There is $0 \neq y \in A$ with $f(y)=r g(y)$ where $r$ is some $n$th root of unity of $R$. Let $R x_{1} \oplus \cdots \oplus R x_{n}$ be a free submodule of $A$ and let $y=x_{1}+\cdots+x_{n}$. There are nonnegative integers $l, l_{1}, \cdots, l_{n}$ with $p^{l} f(y)=r_{y} g(y)$ and $p^{l_{i}} f\left(x_{i}\right)=r_{i} g\left(x_{i}\right)$ for some $r_{y}, r_{1}, \cdots, r_{n} \in R$. Let $m=\max \left\{l, l_{1}, \cdots, l_{n}\right\}$ so that $p^{m} y=p^{m_{x}}+\cdots$ $+p^{m} x_{n}$. Now $f\left(p^{m} y\right)=p^{m-l} p^{l} f(y)=p^{m-l_{r}} g(y)=p^{m-l_{r}} r_{y}\left(g\left(x_{1}\right)+\cdots+g\left(x_{n}\right)\right)$ and $f\left(p^{m} x_{i}\right)=p^{m-l_{i}} p^{l_{i}} f\left(x_{i}\right)=p^{m-l_{i}} r_{i} g\left(x_{i}\right)$. Therefore, $f\left(p^{m} y\right)=p^{m-l_{1}} r_{1} g\left(x_{1}\right)+\cdots+$

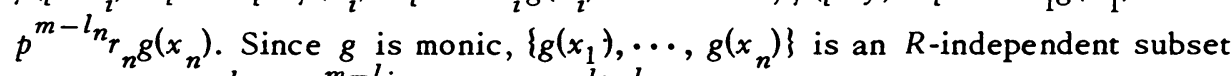
of $A$, so $p^{m-l_{r}}=p^{m-l_{r}}{ }_{i}$ and $r_{i}=p^{l_{i}-l_{r}}$ for $1 \leq i \leq n$.

Let $q=l_{1}+\cdots+l_{n}$. Since $\wedge^{n} f=\bigwedge^{n} g, z=p^{q} f\left(x_{1}\right) \wedge \cdots \wedge f\left(x_{n}\right)=$ $p^{q} g\left(x_{1}\right) \wedge \cdots \wedge g\left(x_{n}\right)$. Moreover, $z=\left(p^{l_{1}} f\left(x_{1}\right)\right) \wedge \cdots \wedge\left(p^{l_{n}} f\left(x_{n}\right)\right)=\left(r_{1} g\left(x_{1}\right)\right) \wedge$ $\cdots \wedge\left(r_{n} g\left(x_{n}\right)\right)=\left(r_{1} r_{2} \cdots r_{n}\right) g\left(x_{1}\right) \wedge \cdots \wedge g\left(x_{n}\right)$. Therefore, $p^{q}=r_{1} r_{2} \cdots r_{n}=$ $p^{l_{1}+l_{2}+\cdots+l_{n}-n l}\left(r_{y}\right)^{n}=p^{q-n l}\left(r_{y}\right)^{n}$, and so $\left(p^{l}\right)^{n}=\left(r_{y}\right)^{n}$. Hence $r_{y}=p^{l} r$, where $r^{n}=$ 1. But $p^{l} f(y)=r_{y} g(y)=p^{l} r g(y)$ so $f(y)=r g(y)$.

Finally, $f-r g$ is an endomorphism of $A$ with nonzero kernel, so $f=r g$ by as sumption.

Corollary 2.3. Assume that $A$ is a totally indecomposable R-module of odd $p$ rank $n$ and that 1 is the only nth root of unity of $R$.

(a) The automorphism group of $A$ is isomorpbic to a subgroup of the automorphism group of $\bigwedge^{n} A$;

(b) $f$ is a unit in $E(A)$ iff $\bigwedge^{n} f$ is a unit in $E\left(\bigwedge^{n} A\right)$;

(c) if $\bigwedge^{n} A$ is reduced, then $E(A)$ is commutative;

(d) if $E\left(\bigwedge^{n} A\right)=R$, then $E(A)=R$.

Proof. (a) Let Aut $(A)$ denote the automorphism group of $A$. Define $p$-det: $\operatorname{Aut}(A) \rightarrow$ Aut $\left(\bigwedge^{n} A\right)$ by $p-\operatorname{det}(f)=\bigwedge^{n} f$. Since $\bigwedge^{n}$ is a functor, $p$-det is a welldefined group homomorphism. If $f \in$ kernel $p$-det, then $\bigwedge^{n} f=1=\bigwedge^{n} 1$. By Theorem 2.2 and Corollary $1.3, f=1$. Thus $p$-det is monic. 
(b) $(\Longrightarrow) \bigwedge^{n}$ is a functor.

$(\Leftarrow)$ Assume $A_{f} \neq 0$. There is $x_{1} \in A \backslash p A$ with $x_{1} \in A_{f}$, since $A_{f}$ is a pure submodule of $A$. Choose $x_{2}, \cdots, x_{n} \in A \backslash p A$ such that $B=R x_{1} \oplus R x_{2} \oplus$ $\cdots \oplus R x_{n}$ is a basic submodule of $\bigwedge^{n} A$. Then $\bigwedge^{n} B=R x_{1} \wedge \cdots \wedge x_{n}$ is a basic submodule of $\bigwedge^{n} A$ by cited fact (4). But $\wedge^{n} f\left(x_{1} \wedge \ldots \wedge x_{n}\right) \in p \wedge^{n} A$ since $f\left(x_{1}\right) \in p A$. This contradicts the assumption that $\Lambda^{n} f$ is a unit.

(c) Since $\bigwedge^{n} A$ is reduced, $\bigwedge^{n} A$ is a p.i. module.

Let $f, g \in E(A)$ and note that

$$
\bigwedge^{n}(f g)=\left(\bigwedge^{n} f\right)\left(\bigwedge^{n} g\right)=\left(\bigwedge^{n} g\right)\left(\bigwedge^{n} f\right)=\stackrel{n}{\Lambda}(g f)^{n}
$$

since $\bigwedge^{n}$ is a functor and $E\left(\bigwedge^{n} A\right)$ is commutative. By Theorem 2.2 and the assumtion that 1 is the only $n$th root of unity, $f g=g f$.

(d) If $\bigwedge^{n} f=r \in R$, then $\bigwedge^{n}\left(f^{n}\right)=\left(\bigwedge^{n} f\right)^{n}=r^{n}=\bigwedge^{n} r$. By Theorem 2.2, $f^{n}=r$. Since Hom $\left(\bigwedge^{n} A, \bigwedge^{n} A\right)=R, \bigwedge^{n} A$ is reduced. By (b) $f$ is a nonunit of $E(A)$ iff $\bigwedge^{n} f$ is a nonunit of $E\left(\bigwedge^{n} A\right)$. Thus $f$ is a nonunit iff $f \in p E(A)$, since $f^{n}=r \in R$.

By (a), the units of $E(A)$ are isomorphic to a subgroup of the units of $\bigwedge^{n} A$, hence a subgroup of the units of $R$. It follows that $E(A)=R$.

3. Examples. We assume, without further comment, the notation and results of [1].

If $A$ is a t.f. $R$-module with $p$-rank $n$, rank $n+k$, and matrix representative $\left(\begin{array}{ll}l & \Gamma \\ 0 & l\end{array}\right)$, then $\wedge^{n} A$ has a standard matrix representative $\left(\begin{array}{ll}l & \Delta \\ 0 & 1\end{array}\right)$. The matrix $\left(\begin{array}{l}\Delta \\ 1\end{array}\right)$ is an $R^{*}$-column matrix with $C_{n+k, n}$ elements, obtained by taking the determinants of the $n \times n$ minors of $\left(\begin{array}{l}r \\ I\end{array}\right)$.

Example 3.1. Given positive integers $n$ and $k$, there is a t.f. $R$-module $A$ with $p$-rank $n$, rank $n+k$ such that $\bigwedge^{n} A$ is reduced.

Proof. Choose $A$ with matrix representative $\left(\begin{array}{ll}I & \Gamma \\ 0 & I\end{array}\right)$, where $\Gamma$ is a $k \times n R^{*}$ matrix and $\left\{\gamma_{i j} \in \Gamma \mid 1 \leq i \leq k, 1 \leq j \leq n\right\}$ is an algebraically independent set over $K$. Let $\left(\begin{array}{ll}l & \Delta \\ 0 & 1\end{array}\right)$ be the standard matrix representative for $\bigwedge^{n} A$. Then $\left(\begin{array}{l}\Delta \\ 1\end{array}\right)$ has $K$-independent rows, by the choice of $\Gamma$, so $\wedge^{n} A$ is reduced.

Example 3.2. Given positive integers $n$ and $k$, there is an indecomposable (in fact, strongly indecomposable) $R$-module. $A$ of $p$-rank $n$, rank $n+k$ such that $A$ is not totally indecomposable. Furthermore, $E(A)=R$.

Proof. We prove the case $n=k=2$ and leave the general case to the reader. Let $V^{*}=K^{*} x_{1} \oplus K^{*} x_{2} \oplus K^{*} x_{3} \oplus K^{*} x_{4}$ be a $K^{*}$-vector space of dimension 4 and choose $A$ with matrix representative $\left(\begin{array}{cc}I & \Gamma \\ 0 & l\end{array}\right)$, where $\Gamma=\left(\begin{array}{cc}a & b \\ 0 & c\end{array}\right),\{a, b, c, 1\} \subset R^{*}$ and $\{a, b, c\}$ is an algebraically indepc ndent set over $K$. In other words, $A=a^{*}(V)$ $\cap \bar{A}$, where $\alpha^{*}(V)=K x_{1} \oplus K x_{2} \oplus K\left(x_{3}+a x_{1}\right) \oplus K\left(x_{4}+b x_{1}+c x_{2}\right)$ and 
$\bar{A}=R^{*} x_{1} \oplus R^{*} x_{2} \oplus K^{*} x_{3} \oplus K^{*} x_{4}$. Then $B=\left(K x_{1} \oplus K\left(x_{3}+a x_{1}\right)\right) \cap\left(R^{*} x_{1} \oplus\right.$ $\left.K^{*} x_{3}\right)$ is a pure submodule of $A$ with $p$-rank 1 , rank 2 . Consequently, $A$ is not totally indecomposable by Theorem 1.1 .

The standard matrix representative of $\bigwedge^{2} A$ is $\left(\begin{array}{ll}I & \Delta \\ 0 & 1\end{array}\right)$, where $\Delta$ is the transpose of $(a c,-b, a,-c, 0)$. Thus, $\bigwedge^{2} A=D \oplus K$, where $D$ is reduced. If $A=$ $B \oplus C$, then a comparison of ranks and $p$-ranks shows that $r(B)=r(C)=2$ and $r p(B)=r p(C)=1$. Thus $\bigwedge^{2} A \simeq \bigwedge^{2} B \oplus(B \otimes C) \simeq \bigwedge^{2} C \oplus K \oplus(B \otimes C) \oplus K$, a contradiction to the preceding remarks. Therefore, $A$ is indecomposable.

The proof that $A$ is strongly indecomposable follows from the observation that exterior powers preserve quasi-isomorphism and from the preceding argument.

A persistent reader may prove that if $f \in E(A)$ and if $f^{*}: V^{*} \quad V^{*}$ is the unique extension of $f$, then $f^{*}\left(a^{*}(V)\right) \subset a^{*}(V), f^{*}\left(K^{*} x_{3} \oplus K^{*} x_{4}\right) \subset K^{*} x_{3} \oplus K^{*} x_{4}$ and $\{a, b, c\}$ algebraically independent imply that $f^{*}$ is multiplication by some $r \in R$.

Example 3.3. There is a totally indecomposable $R$-module $A$ of $p$-rank 2, rank 4 such that $\bigwedge^{2} A$ is not reduced.

Proof. Let $A$ be a t.f. $R$-module with matrix representative $\left(\begin{array}{ll}I & \Gamma \\ 0 & I\end{array}\right)$, where $\Gamma=$ $\left(\begin{array}{ll}a & b \\ b & d\end{array}\right)$ and $\{a, b, d\}$ is a subset of $R^{*}$ which is algebraically independent over $K$. Then $\bigwedge^{2} A$ has $\left(\begin{array}{ll}I & \Delta \\ 0 & 1\end{array}\right)$ as a standard matrix representative, where $\Delta$ is the transpose of $\left(a d-b^{2},-b, a,-d, b\right)$. Consequently, $\wedge^{2} A$ is not reduced, since $\left\{a d-b^{2}\right.$, $-b, a,-d, b\}$ is a $K$-dependent subset of $R^{*}$.

Assume that $A$ is not totally indecomposable. By Theorem 1.1, there is a nonfree pure submodule $C$ of $A$ with $r p(C)<2$. Since $A$ is reduced of rank 4 and $p$-rank 2 , then $r(C)=2$ or $r(C)=3$. Let $x$ and $y$ be two $R$-independent elements of $C$ and let $B$ be the pure submodule of $C$ generated by $x$ and $y$. Then $B$ is a pure submodule of $A$ with $p$-rank 1 and rank 2 . Hence if $A$ has no pure submodules with $p$-rank 1 and rank 2 , then $A$ is totally indecomposable.

By the preceding remarks, it suffices to prove that if $B$ is a t.f. $R$-module of $p$-rank 1 , rank 2 and if $0 \neq f \in \operatorname{Hom}(B, A)$, then $f$ is not monic. Let $\left(\begin{array}{ll}1 & e \\ 0 & 1\end{array}\right)$ be a matrix representative for $B$. Then $B=\beta^{*}(U) \cap \bar{B}$, where $\beta^{*}(U)=K z_{1} \oplus$ $K\left(z_{2} \oplus e z_{1}\right)$ and $\bar{B}=R^{*} z_{1} \oplus K^{*} z_{2} \subset U^{*}=K^{*} z_{1} \oplus K^{*} z_{2}$. Moreover, $A=\alpha^{*}(V) \cap \bar{A}$, where $a^{*}(V)=K x_{1} \oplus K x_{2} \oplus K\left(x_{3}+a x_{1}+b x_{2}\right) \oplus K\left(x_{4}+b x_{1}+d x_{2}\right)$ and $\bar{A}=R^{*} x_{1}$ $\oplus R^{*} x_{2} \oplus K^{*} x_{3} \oplus K^{*} x_{4} \subset V^{*}=K^{*} x_{1} \oplus K^{*} x_{2} \oplus K^{*} x_{3} \oplus K^{*} x_{4}$. The map $f: B \rightarrow A$ has a unique extension $f^{*}: U^{*} \rightarrow V^{*}$ with $f^{*}(\bar{B}) \subset \bar{A}, f^{*}\left(K^{*} z_{2}\right) \subset K^{*} x_{3} \oplus K^{*} x_{4}$ and $f a^{*}(V) \subset \beta^{*}(U)$. One can now show that $f\left(z_{2}\right) \in K x_{3} \oplus K x_{4}$ and that $e \in R$, since $\{a, b, d\}$ is algebraically independent over $K$. Thus, $B \simeq R \oplus K$. Since $A$ is reduced, $K \subset \operatorname{ker} f$ and so $f$ is not monic.

Example 3.4. There are t.f. $R$-modules $A$ and $B$ of $p$-rank 2 , rank 4 such that $\bigwedge^{2} A$ is reduced, $\bigwedge^{2} A \simeq \bigwedge^{2} B$, and the modules $A$ and $B$ are not quasiisomorphic. 
Proof. Choose $A$ and $B$ with matrix representatives $\left(\begin{array}{ll}I & \Gamma \\ 0 & I\end{array}\right)$ and $\left(\begin{array}{ll}I & \Delta \\ 0 & I\end{array}\right)$, respectively, where $\Gamma=\left(\begin{array}{ll}a & b \\ c & d\end{array}\right), \Delta=\left(\begin{array}{ll}d & b \\ c & a\end{array}\right)$ and $\{a, b, c, d\}$ is a subset of $R^{*}$, algebraically independent over $K$. Since $\Lambda^{2} A$ and $\Lambda^{2} B$ have identical standard matrix representatives, $\wedge^{2} A \simeq \bigwedge^{2} B$. The fact that $A$ and $B$ are not quasi-isomorphic follows from the fact that $\{a, b, c, d\}$ is an algebraically independent set over $K$.

Note that if $F$ is the duality given in [1], then $B=F A$.

Example 3.5. There is a totally indecomposable $R$-module $A$ of $p$-rank 3, rank 4 such that $E(A)$ is not local.

Proof. Let $R$ be the localization of the ring of integers at a prime $p>3$, and let $f(X)=X^{4}+p X^{3}+p X^{2}+(1-p) X+p$. Then $f(-1) \equiv 0(\bmod p)$ and $f^{\prime}(-1) \not \equiv$ $0(\bmod p)$ where $f^{\prime}(X)=4 X^{3}+3 p X^{2}+2 p X+(1-p)$ is the derivative of $f$. Therefore, $f(X)$ has a root $a$ in $R^{*}$ (e.g., see Bachman [2]). In fact, $a=-1+p b$, for some $b \in R^{*}$, so $a$ is a unit in $R^{*}$.

Choose $A$ with matrix representative $\left(\begin{array}{ll}1 & \Gamma \\ 0 & I\end{array}\right)$, where $\Gamma=\left(a, a^{2}, a^{3}\right)$, i.e. $A=$ $a^{*}(V) \cap \bar{A} \subset V^{*}$, where $V^{*}=K^{*} x_{1} \oplus K^{*} x_{2} \oplus K^{*} x_{3} \oplus K^{*} x_{4}, a^{*}(V)=K x_{1} \oplus K x_{2}$ $\oplus K x_{3} \oplus K y, y=x_{4}+a x_{1}+a^{2} x_{2}+a^{3} x_{3}$, and $\bar{A}=R^{*} x_{1} \oplus R^{*} x_{2} \oplus R^{*} x_{3} \oplus K^{*} x_{4}$.

Define $g: V^{*} \rightarrow V^{*}$ by

$$
\begin{aligned}
& g\left(x_{1}\right)=x_{1}+p x_{2}+\left(-p+p^{2}\right) x_{3}, \\
& g\left(x_{2}\right)=(p+p a) x_{1}+\left(1+p a^{2}\right) x_{2}+\left(-p^{2}+p+p a^{3}\right) x_{3}+p x_{4}, \\
& g\left(x_{3}\right)=a x_{1}+\left(p+a^{2}\right) x_{2}+\left(1-p^{2}+a^{3}\right) x_{3}+x_{4}, \\
& g\left(x_{4}\right)=\left(p-a^{2} p-a^{3}\right) x_{4} .
\end{aligned}
$$

Then

$$
g(y)=(-p+p a) x_{1}+p a^{2} x_{2}+\left(-p^{2}+p a^{3}\right) x_{3}+p x_{4},
$$

so $g\left(\alpha^{*}(V)\right) \subset \alpha^{*}(V)$ and $g(\bar{A}) \subset \bar{A}$. Therefore, $g$, restricted to $A$, is an endomorphism of $A$.

Observe that $g(y) \in p A$ and that $y \in A \backslash p A$ since $a$ is a unit in $R^{*}$. Thus $g$ is not a unit of $E(A)$ since $A_{g} \neq 0$. Furthermore, $(1-g)\left(x_{1}\right)=-p x_{2}-$ $\left(p^{2}-p\right) x_{3} \in p A$ and $x_{1} \in A \backslash p A$ so $1-g$ is not a unit of $E(A)$. This proves that $E(A)$ is not a local ring.

Finally, we prove that $A$ is totally indecomposable. Note that

$$
f(X) \equiv X^{4}+X^{3}+X^{2}+1 \equiv(X+1)\left(X^{3}+X+1\right) \quad(\bmod 2) .
$$

If $f(X)$ is reducible in $Z[X]$, then $f(X)$ is the product of a linear factor and a cubic factor since $X^{3}+X+1$ is irreducible modulo 2. Clearly, $f(X)$ has no linear factors in $Z[X]$, so $f(X)$ is irreducible in $Z[X]$, hence $Q[X]$, where $Q$ is the field of rational numbers. Therefore, $f(X)$ is an irreducible polynomial in 
$R[X]$ and $\left\{1, a, a^{2}, a^{3}\right\}$ is an $R$-independent set. It now follows that $\bigwedge^{3} A$ is reduced since the standard matrix representative of $\wedge^{3} A$ is $\left(\begin{array}{ll}I & \Delta \\ 0 & 1\end{array}\right)$ where $\Delta$ is the transpose of $\left(a^{3}, a,-a^{2}\right)$.

\section{REFERENCES}

1. D. Arnold, A duality for torsion free modules of finite rank over a discrete valuation ring, Proc. London Math Soc. (3) 24 (1972), 204-216.

2. G. Bachman, Introduction to p-adic numbers and valuation theory, Academic Press, New York, 1964. MR 30 \#90.

3. N. Bourbaki, Éléments de mathématique. I: Les structures fondamentales de l'analyse. Fasc. VII. Livre II: Algèbre. Chap. 3. Algèbre multilinéaires, 2nd ed., Actualités Sci. Indust., no. 1044, Hermann, Paris, 1958. MR 30 \#3104.

4. H. Flanders, Tensor and exterior powers, J. Algebra 7(1967), 1-24. MR 35 \#2919.

5. L. Fuchs, Infinite abelian groups. Vol. 1, Pure and Appl. Math., vol. 36, Academic Press, New York, 1970. MR 41 \#333.

6. - Abelian groups, Akad. Kiadó, Budapest, 1958. MR 21 \#5672.

7. I. Kaplansky, Infinite abelian groups, Univ. of Michigan Press, Ann Arbor, Mich., 1954. MR 16, 444.

8. J. Lambek, Lectures on rings and modules, Blaisdell, Waltham, Mass., 1966. MR 34 \#5857.

9. J. Rotman, A note on completions of modules, Proc. Amer. Math. Soc. 11(1960), 356-360. MR 22 \#5631.

DEPARTMENT OF MATHEMATICAL SCIENCES, NEW MEXICO STATE UNIVERSITY, LAS CRUCES, NEW MEXICO 88001 